\title{
Low Levels of MicroRNA-10a in Cardiovascular Endothelium and Blood Serum Are Related to Human Atherosclerotic Disease
}

\author{
Jong-Tar Kuo $\mathbb{D}^{1},{ }^{1}$ Hsiao-En Tsai $\mathbb{D}^{2,3}$ Ching-Ting Lin ${ }^{2},{ }^{4}$ Chih-I Lee, ${ }^{5}$ Pei-Ling Lee, ${ }^{5}$ \\ Yu-Rong Ruan, ${ }^{1}$ Jeng-Jiann Chiu, ${ }^{5}$ and Ding-Yu Lee $\mathbb{D}^{1}$ \\ ${ }^{1}$ Department of Biological Science and Technology, China University of Science and Technology, Taipei, Taiwan \\ ${ }^{2}$ Division of Cardiovascular Surgery, Department of Surgery, National Taiwan University Hsin-Chu Hospital, Hsinchu, Taiwan \\ ${ }^{3}$ Graduate Institute of Clinical Medicine, National Taiwan University College of Medicine, Taipei, Taiwan \\ ${ }^{4}$ School of Chinese Medicine, China Medical University, Taichung, Taiwan \\ ${ }^{5}$ Institute of Cellular and System Medicine, National Health Research Institutes, Miaoli, Taiwan \\ Correspondence should be addressed to Ding-Yu Lee; leedingyu1215@gmail.com
}

Jong-Tar Kuo, Hsiao-En Tsai, and Ching-Ting Lin contributed equally to this work.

Received 22 April 2021; Accepted 30 June 2021; Published 15 July 2021

Academic Editor: Michael S. Wolin

Copyright (c) 2021 Jong-Tar Kuo et al. This is an open access article distributed under the Creative Commons Attribution License, which permits unrestricted use, distribution, and reproduction in any medium, provided the original work is properly cited.

\begin{abstract}
Background. MicroRNA-10a (miR-10a) inhibits transcriptional factor GATA6 to repress inflammatory GATA6/VCAM-1 signaling, which is regulated by blood flow to affect endothelial function/dysfunction. This study aimed to identify the expression patterns of miR-10a/GATA6/VCAM-1 in vivo and study their implications in the pathophysiology of human coronary artery disease (CAD), i.e., atherosclerosis. Methods. Human atherosclerotic coronary arteries and nondiseased arteries were used to detect the expressions of miR-10a/GATA6/VCAM-1 in pathogenic vs. normal conditions. In addition, sera from CAD patients and healthy subjects were collected to detect the level of circulating miR-10a. Results. The comparison of human atherosclerotic coronary arteries with nondiseased arteries demonstrated that lower levels of endothelial miR-10a are related to human atherogenesis. Moreover, GATA6/VCAM-1 (a downstream target of miR-10a) was highly expressed in the endothelium, accompanied by the reduced levels of miR-10a during the development of human atherosclerosis. In addition, CAD patients had a significantly lower concentration of miR-10a in their serum compared to healthy subjects. Conclusions. Our findings suggest that low miR-10a and high GATA6/VCAM-1 in the cardiovascular endothelium correlates to the development of human atherosclerotic lesions, suggesting that miR-10a signaling has the potential to be developed as a biomarker for human atherosclerosis.
\end{abstract}

\section{Introduction}

Vascular endothelial cells (ECs) modulated by shear stress resulting from blood flow play vital roles in cardiovascular pathology [1]. The blood shear stresses, i.e., oscillatory shear (OS) and pulsatile shear stress (PS), activate different mechanotransduction pathways to modulate EC dysfunction and function [1]. OS occurs in bifurcation and curvature sites (the atherosclerosis-susceptible regions) of the cardiovascular system and serves as "pathogenic shear" to induce EC inflammatory signaling and promote the development of atherosclerosis. In contrast, PS occurs in straight regions (the atherosclerosis-resistant regions) of the cardiovascular system and serves as a "protective shear" to inhibit EC inflammatory signaling and protect blood vessels from atherosclerosis [1-4].

MicroRNAs (miRs) are the noncoding RNA molecules that bind to the $3^{\prime}$-untranslated regions $\left(3^{\prime}\right.$-UTR) of the target mRNAs and mediate RNA silencing and regulate gene expression. Reportedly, miRs regulate several biological processes, including regulating cardiovascular functions [5-7]. Moreover, studies have shown that hemodynamic shear stress regulates the expressions of miRs promoting EC function or dysfunction; in particular, OS-modulated miRs 
promote the development of atherosclerosis [5, 8-10]. An miR microarray study in an in vivo experimental swine model demonstrated that EC miR-10a is a vital shear-regulated $\mathrm{miR}$ with the lowest relative expression among all miRs in the pathogenic OS region (atherosclerosis-susceptible regions) of the cardiovascular system [11]. Moreover, the potential of miR-10a to inhibit inflammatory signaling in ECs is also evident from in vitro and in vivo studies $[11,12]$. In vitro studies have demonstrated that the activation of NF$\kappa \mathrm{B}$ and the expression of inflammatory molecules are induced by the inhibition of EC miR-10a [11]. Furthermore, in a previous study, we have demonstrated that miR-10a directly binds to the $3^{\prime}$-UTR of GATA6 (an inflammatory transcriptional factor) to abolish its expression, consequently inhibiting the downstream vascular cell adhesion molecule (VCAM-1) [12]. The study has also reported that PS induces miR-10a expression to repress inflammatory GATA6/VCAM-1 signaling in ECs, whereas OS inhibits miR-10a expression to enhance inflammatory GATA6/ VCAM-1 signaling [12]. Taken together, these studies suggest that the hemodynamic shear stresses, i.e., PS and OS, have differential effects on the alteration of miR-10a expression to turn on the anti-inflammatory and inflammatory signaling pathways, respectively [12]. Recently, we used an ApoE knockout (ApoE-/-) mouse model to show that endothelial miR-10a can be induced by precursor miR-10a or $\operatorname{RAR} \alpha / \operatorname{RXR} \alpha$ agonists to inhibit GATA6/VCAM-1 signaling, inflammatory cell infiltration, and atherosclerotic lesion formation [12-14]. Despite several in vitro and in vivo reports on the anti-inflammatory effect of shear-sensitive miR10a in ECs, the role of endothelial miR-10a/GATA6/VCAM1 signaling during the progression of human atherosclerosis remains unclear in vivo. In addition, the clinical applications of miR-10a in human atherosclerotic disease still need to be identified.

This present study aims to identify the expression patterns of miR-10a/GATA6/VCAM-1 in vivo and study their implications in the pathophysiology of human coronary artery disease (CAD). Here, we compared the diseased human coronary arteries with nondiseased human arteries, i.e., human coronary arteries with advanced atherosclerotic lesion vs. human coronary arteries with minimal atherosclerotic lesion or nondiseased human internal thoracic aorta, to identify the in vivo role of miR-10a/GATA6/ VCAM-1 signaling in pathogenic vs. normal condition in the human cardiovascular system. Furthermore, we correlated expression patterns of miR-10a and GATA6/VCAM-1 in the endothelium with the development of human atherosclerotic lesions. In addition, we investigated the relative levels of miR-10a in the sera collected from human blood from patients with $\mathrm{CAD}$ and healthy subjects.

\section{Materials and Methods}

2.1. Diseased Human Coronary Arteries and Nondiseased Human Arteries. Eight diseased human coronary arteries were harvested from five end-stage heart failure patients undergoing heart transplantation at Tri-Service General Hospital in Taiwan. The study was carried out in accordance with the Declaration of Helsinki and approved by the Institutional Review Board of the Tri-Service General Hospital. Informed consent was obtained from all participants. These arteries contained various stages of atherosclerotic lesions, from minimal lesions (mini. lesion, with no intimal thickening and intimal macrophages) to advanced lesions (advan. lesion, with increased intima thickness and intimal macrophages). Nondiseased internal thoracic arteries obtained from four CAD patients undergoing coronary artery bypass graft surgery (CL artery) were used as control arteries.

\subsection{Detection of miR-10a, GATA6, and VCAM-1 Expressions.} Coronary arteries with advan. lesions and mini. lesions and CL arteries were fixed in formalin and embedded in paraffin blocks. The expression of miR-10a in the cross-sections of these samples was estimated by $\mathrm{miR}$ in situ hybridization. Furthermore, we estimated the expression levels of GATA6 and VCAM-1 by immunohistochemical staining.

\subsection{1. $\mathrm{MiR}$ In Situ Hybridization to Detect miR-10a} Expression. The miR-10a expression in the samples was determined by fluorescence in situ hybridization [15], as described previously [13]. In brief, cross-sections of coronary arteries with advan. lesions and coronary arteries with mini. lesions or nondiseased arteries were incubated with 5'DIG-labeled locked-nucleic acid probes $(20 \mathrm{nmol} / \mathrm{L})$ (Thermo Scientific, MA) at an optimal temperature. The cross-sections were further incubated with HRP-conjugated antibody against DIG (Roche Applied Science, Germany), and the signal was amplified using TMR-tyramide (Perkin Elmer, MA). The slides were mounted, and the images were captured using a fluorescence microscope.

\subsubsection{Immunohistochemical Staining to Detect the Expres-} sions of GATA6 and VCAM-1. Immunohistochemical staining was performed as previously described [13]. Two serial sections were subjected to immunohistochemical staining for detecting GATA6 or VCAM-1 and the EC marker (i.e., von Willebrand factor ( $\mathrm{VWF})$ ), respectively. In brief, serial sections ( $4 \mu \mathrm{m}$ thick) of coronary and internal thoracic arteries were deparaffinized and then blocked for $1 \mathrm{~h}$ with serum albumin dissolved in phosphate-buffered saline $(5 \mathrm{mg} / \mathrm{mL})$. One section was incubated with antiGATA6 antibody (Santa Cruz, CA) or anti-VCAM-1 antibody (Santa Cruz, CA) (1:50) for $1 \mathrm{~h}$ at $37^{\circ} \mathrm{C}$, followed by rhodamine-conjugated secondary antibody $(1: 1000)$ in the presence of DAPI for $2 \mathrm{~h}$ at room temperature. The second section was incubated with an anti-vWF antibody (Santa Cruz, CA) (1:50), followed by the secondary antibody for $2 \mathrm{~h}$ at room temperature. The slides were mounted, and the images were captured using a fluorescence microscope.

\subsection{Blood Serum Collection, RNA Extraction, and miR-10a} Detection. The serum level of miR-10a was assessed following the procedures described in a previous study [13]. Fresh blood from human subjects without $(n=13)$ or with $(n=30)$ CAD was collected into $10 \mathrm{~mL}$ BD Vacutainer 
K3EDTA tubes (BD Biosciences, MA). The study and experimental design was approved by the Ethics Review Board of the National Health Research Institutes, and informed consent was obtained from all participants. Blood serum was collected by centrifugation at $2500 \mathrm{x}$ g for $10 \mathrm{~min}$ at room temperature and then stored at $-80^{\circ} \mathrm{C}$. Total RNA was further isolated using TRIzol reagent (Thermo Scientific) and supplemented with $5 \mathrm{nmol} / \mathrm{L}$ Caenorhabditis elegans miR-39 (cel-miR-39) (Thermo Scientific) as Spike-in Control, as per the protocol described previously [16]. The levels of miR-10a in these sera were measured using RT-qPCR with iQ SYBR Green supermix (Bio-Rad).

\section{Results}

3.1. Low miR-10a Level in the Endothelium Is Related to the Progression of Atherosclerotic Lesions in Human Coronary Arteries In Vivo. The results of $\mathrm{miR}$ in situ hybridization show pronounced staining of miR-10a in the endothelium of nondiseased CL artery (Figure 1, top row) and coronary arteries with mini. lesion (Figure 1, middle row). However, the expression of miR-10a was rare in the endothelium of diseased coronary arteries with advan. lesion (Figure 1, bottom row). These results suggest that low miR-10a levels in the endothelium could be related to the development of atherosclerotic lesions in human coronary arteries in vivo.

\subsection{GATA6 (Direct Target of miR-10a) Is Highly Expressed in} the Endothelium of Human Atherosclerotic Coronary Arteries In Vivo. Our previous in vitro study demonstrated that the transcriptional factor GATA6 is the direct target of miR-10a, and that its expression can be inhibited by miR-10a through its binding to the $3^{\prime}$-UTR of GATA6 [12]. Here, we used immunohistochemical staining to investigate the in vivo expression patterns of GATA6 in the endothelia of nondiseased human arteries and diseased human coronary arteries. As shown in Figure 2, GATA6 is highly expressed in the endothelium of diseased coronary arteries with advan. lesions (Figure 2, bottom row). In contrast, the expression of GATA6 was not detected in the endothelium of the CL artery (Figure 2, top row) and coronary arteries with mini. lesions (Figure 2, middle row). These results suggest that GATA6, the direct target of miR-10a is highly expressed in the endothelium of human atherosclerotic coronary arteries in vivo.

\subsection{VCAM-1 (a Downstream Target of miR-10a) Is Upregu-} lated in the Endothelium of Human Atherosclerotic Coronary Arteries In Vivo. The inflammatory molecule, VCAM-1, regulated transcriptionally by the transcriptional factor GATA6, has been identified as a downstream molecule of miR-10a and GATA6 $[12,17]$. In this study, we showed that the expression of VCAM-1 was upregulated in the endothelium of diseased coronary arteries with advan. lesions (Figure 3, bottom row), whereas it was downregulated in the endothelium of the CL arteries (Figure 3, top row) and coronary arteries with mini. lesions (Figure 3, middle row). Furthermore, the expression patterns of VCAM-1 in the

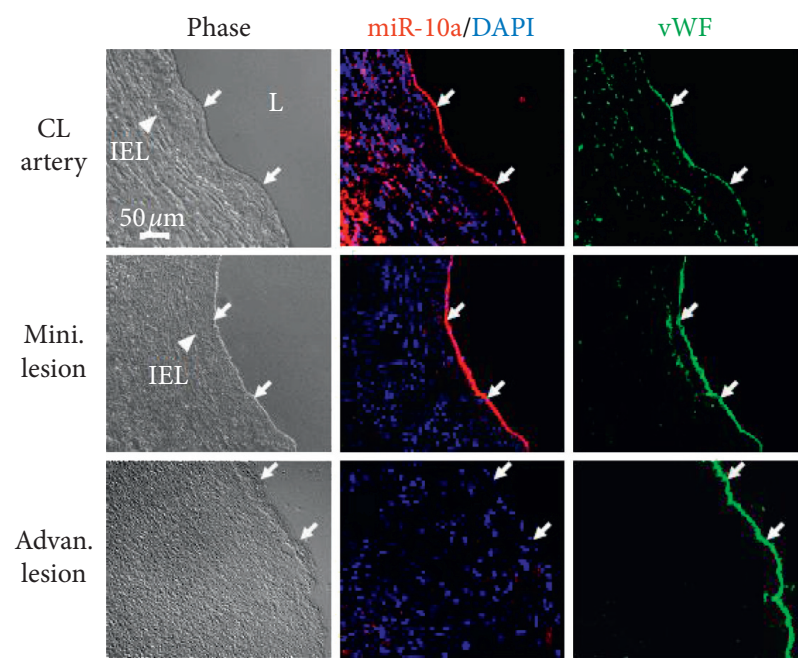

FIGURE 1: miR-10a is highly expressed in the EC layer of human nondiseased arteries but is decreased in atherosclerotic coronary arteries. Cross-section samples of coronary arteries with advanced atherosclerotic lesion (advan. lesion) vs. coronary arteries with minimal atherosclerotic lesion (mini. lesion) or nondiseased internal thoracic aorta (CL artery) were stained with human miR-10a and vWF (i.e., EC marker) and counterstained with DAPI. vWF was used to identify the endothelium of blood vessels. Arrow, vWFpositive ECs; IEL, internal elastic lamina; $L$, lumen; EC, endothelial cell; CL artery, control artery.

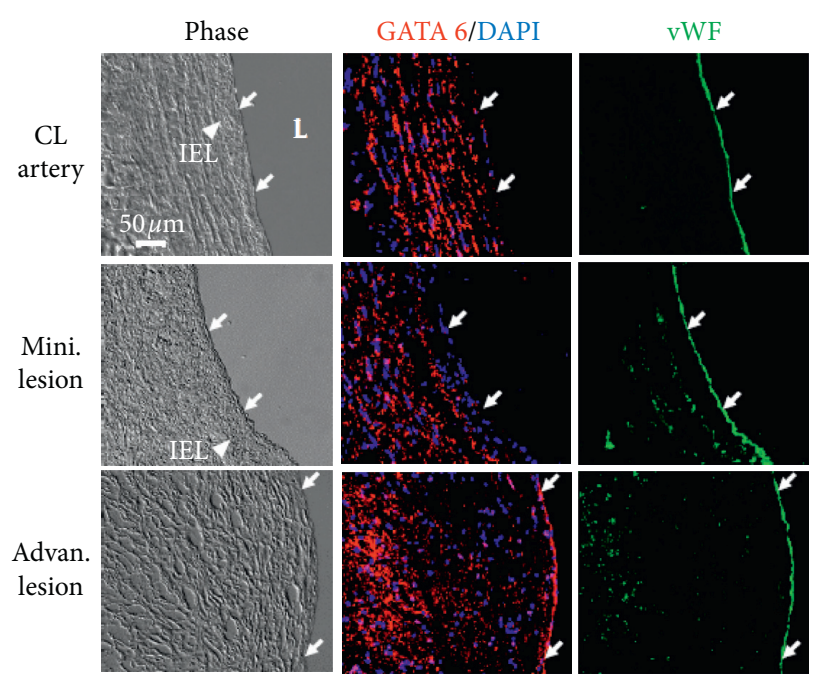

FIGURE 2: GATA6 (direct target of miR-10a) in the endothelium is highly expressed in human atherosclerotic coronary arteries in vivo. Cross-sections of diseased human arteries vs. nondiseased human arteries, i.e., coronary arteries with advan. lesions vs. coronary arteries with mini. lesions or CL arteries, were immunohistochemically stained to detect inflammatory transcriptional factor GATA6 and vWF and counterstained with DAPI. vWF was used to identify the endothelium of blood vessels. Arrow, vWF-positive endothelial cells; IEL, internal elastic lamina; L lumen; CL artery, control artery.

endothelium of nondiseased and diseased arteries were similar to those of GATA6, implying that the expression of inflammatory molecule VCAM-1 is also upregulated in the endothelium of human atherosclerotic coronary arteries in vivo. 


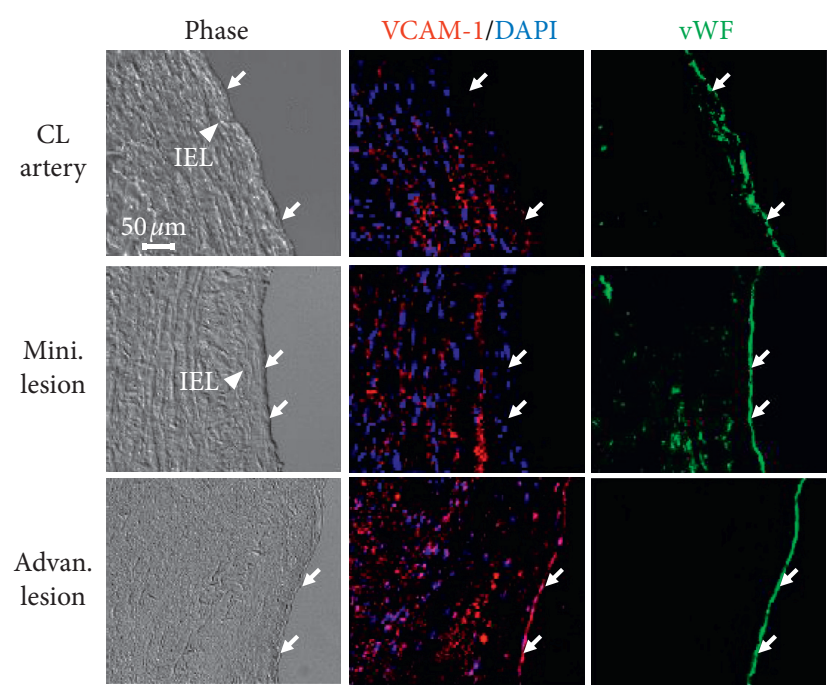

FIGURE 3: miR-10a downstream VCAM-1 in the endothelium is upregulated in human atherosclerotic coronary arteries in vivo. Cross-sections of diseased coronary arteries with advan. lesions vs. coronary arteries with mini. lesions or CL arteries were immunohistochemically stained for the inflammatory molecule VCAM-1 and $\mathrm{vWF}$ and counterstained with DAPI. vWF was used to identify the endothelium of blood vessels. Arrow, vWF-positive endothelial cells; IEL, internal elastic lamina; L lumen; CL artery, control artery.

\subsection{Low Level of miR-10a in Blood Serum Is Associated with the} Development of Human Atherosclerosis. Circulating $\mathrm{miR}$ in the blood serum has been identified as a diagnostic component in atherosclerotic disease [16]. Here, we collected blood samples from patients with CAD and healthy subjects to detect the level of miR-10a in their sera. The characteristics of patients with CAD and healthy subjects are shown in Table S1. Results showed that miR-10a level in the blood serum of patients with CAD was significantly lower than that in healthy subjects (Figure 4), indicating that low circulating miR-10a level in the blood serum is associated with the development of human atherosclerosis.

\section{Discussion}

In the present study, we showed that low miR-10a in both cardiovascular endothelium and blood serum is highly related to the development of CAD, i.e., atherosclerosis, and can be used as a potential biomarker for human atherosclerosis, as evident from the findings of this study. First, miR in situ hybridization results demonstrated that miR-10a is highly expressed in the endothelial layer of CL arteries and coronary arteries with mini. lesions, but not in the endothelial layer of coronary arteries with advan. lesions. Second, in the endothelial layer of nondiseased human arteries, the lack of transcriptional factor GATA6, which is the direct target of miR-10a, was accompanied by the high level of miR-10a. In contrast, the intense expression of GATA6 in the endothelial layer of diseased coronary arteries was accompanied by the low expression of miR-10a. Third, expression of the inflammatory molecule, VCAM-1, which is the downstream target of miR-10a and GATA6, was downregulated in the endothelial layer of nondiseased

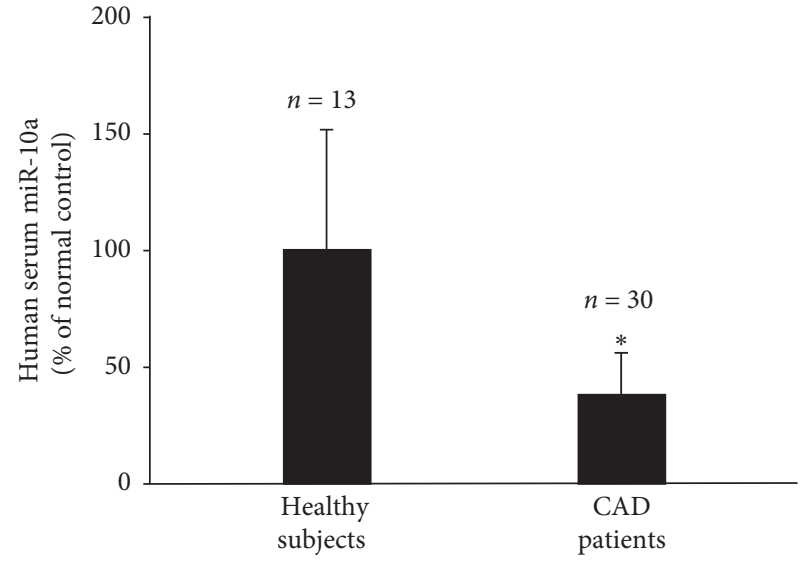

Figure 4: Low level of miR-10a in blood serum is highly related to the progression of human atherosclerotic disease. The levels of miR-10a in blood serum from human subjects without $(n=13)$ or with CAD $(n=30)$ were detected by qPCR. Data show the mean $\pm S D$ and are presented as the percentage changes compared to the healthy control groups. ${ }^{*}, P<0.05$ vs. healthy subjects. $C A D$, coronary artery disease.

arteries, whereas it was upregulated in the endothelial layer of diseased coronary arteries. Fourth, detection of miR-10a in human blood from patients with CAD compared to that from healthy subjects demonstrated that miR-10a level in patient serum is significantly lower than that in serum from healthy subjects. Collectively, our findings provide a new concept that the reduced expression of miR-10a in both cardiovascular endothelium and blood serum may promote human blood vessels toward atherosclerotic lesions in the cardiovascular system.

4.1. Low Level of "Atheroprotective miR-10a" in the Endothelium Is Highly Related to the Development of Human CAD, i.e., Atherosclerosis. MiRs have emerged as vital factors affecting cardiovascular pathology [5-8]. Several miRs can be modulated by blood shear to regulate signaling molecules involved in signal transductions that modulate vascular EC dysfunctions, i.e., inflammation, proliferation, and oxidation [5, 8-10]. Notably, Fang et al. used miR microarray to analyze the expressions of various miRs in the atherosclerosis-susceptible region (OS region) relative to those in atherosclerosis-resistant regions (PS region) of the cardiovascular system in an animal swine model and reported that miR-10a was the least expressed miR in the endothelium of OS region, i.e., aortic arch and aorta-renal branches, compared to that in other regions in vivo [11]. Furthermore, they also transfected human umbilical vein endothelial cells (HUVEC) with antagomiR-10a and demonstrated that miR10 a exerts an anti-inflammatory effect by inhibiting NF- $\kappa \mathrm{B}$ signaling in ECs [11]. Our previous studies used an in vitro flow system to identify that proatherogenic OS induces a sustained decrease in miR-10a expression, whereas atheroprotective PS induces a sustained increase in miR-10a expression in ECs. By investigating the effect of fluid shear on all the examined shear-sensitive miRs in ECs, our results also showed miR-10a as the OS-modulated miR with the 
lowest expression [12]. We further used an in vivo diseased ApoE-/- mouse model to demonstrate that endothelial miR$10 \mathrm{a}$ is increased in the PS region of the cardiovascular system but inhibited in the OS region. These findings indicated that the precursor miR-10a injection could induce miR-10a expression in the endothelium of blood vessels to inhibit atherosclerotic lesion formation in ApoE-/- mice [12]. In accordance, the present findings demonstrated that miR-10a is undetectable in the endothelium of atherosclerotic lesions in diseased human coronary arteries, in contrast to its high expression in the endothelium of nondiseased arteries. These findings suggest that the low level of "atheroprotective miR$10 \mathrm{a}$ " in the endothelium is highly related to the development of human CAD, i.e., atherosclerosis.

4.2. Low Level of Endothelial miR-10a Results in an Intense Expression of Transcriptional Factor GATA6 during the Development of Human Atherosclerosis. GATA6 has been identified as the direct target of miR-10a, and its expression can be inhibited by miR-10a binding to 3'-UTR of GATA6 [12]. GATA factors are zinc finger-containing transcriptional factors that bind specific DNA region in the promoter to regulate gene expression [18]. GATA6 is a member of the GATA factor family. It is expressed in a wide range of human tissues, e.g., heart, liver, kidney, pancreas, and spleen, to regulate differential responses in these tissues [19]. In ECs, GATA6 has been defined as the vital transcriptional factor for inflammatory signaling [17]. Our previous study has shown that the expression of GATA6 can be modulated by shear-regulated miR-10a in the ECs. Atheroprotective PS induces miR-10a to inhibit the expression of its direct target GATA6, whereas atherogenic OS represses miR-10a to turn on the expression of GATA6 in the ECs in vitro [12]. In vivo results using ApoE-/- mice showed that GATA6 is overexpressed in the endothelium of atherosclerosis-susceptible region (OS region) of the cardiovascular system, but not in the endothelium of atherosclerosis-resistant region (PS region). Injection of ApoE-/- mice with miR-10a precursor inhibited the GATA6 expression in the endothelium of atherosclerosis-susceptible region [12]. The present study further extended these findings in the development of human atherosclerotic disease. The present study demonstrated that the inhibition of GATA6 is accompanied by the high expression of miR-10a in the endothelium of human nondiseased arteries. In contrast, the intense expression of GATA6 is accompanied by the reduced expression of miR$10 \mathrm{a}$ in the endothelium of human diseased coronary arteries. Taken together, our findings suggest that a low level of endothelial miR-10a results in an intense expression of GATA6 during the development of human atherosclerotic disease.

4.3. Low miR-10a Level Induces Transcriptional Factor GATA6 to Upregulate Inflammatory VCAM-1 during the Development of Human Atherosclerosis. Cell adhesion molecules are involved in cell-cell interaction [20]. In atherogenesis, ECs induce the expression of cell adhesion molecule, i.e., VCAM-1, to facilitate the adherence of monocytes to the endothelium as well as their infiltration into the vessel wall at the initiation of atherosclerosis [21]. Thus, VCAM-1 is an important cell adhesion molecule for the initiation of atherosclerosis [21, 22]. VCAM-1 has been identified to be regulated transcriptionally by the transcriptional factor GATA6 in ECs in response to TNF $\alpha$, and its promoter region contains the GATA6-binding site $[17,23]$. Our previous results have shown that VCAM-1 can be regulated transcriptionally by GATA6 in ECs in response to blood fluid flow. Moreover, PS and OS can alter EC miR10a levels to modulate the expression of GATA6 and subsequently regulate the expression of its downstream molecule VCAM-1 in vitro [12]. Our in vivo studies on the diseased ApoE-/- mouse model showed that VCAM-1 is upregulated in the endothelium of the OS region in the cardiovascular system but not in that of the PS region. Furthermore, it has also been shown that endothelial miR10a induction by injection of miR-10a precursor can inhibit GATA6/VCAM-1 signaling [12] and inflammatory cell infiltration in the atherosclerotic vessel wall in ApoE-/- mice [13]. In the present study, we found VCAM-1 to be upregulated in the endothelium of human diseased coronary arteries but downregulated in that of human nondiseased arteries. The expression patterns of VCAM-1 in human nondiseased arteries vs. human diseased coronary arteries were similar to those of the transcriptional factor GATA6. Taken together, these results imply that a low miR-10a level induces an intense expression of the transcriptional factor GATA6 to upregulate the expression of the inflammatory molecule VCAM-1 during the development of human atherosclerotic disease.

4.4. Low Levels of Circulating miR-10a in Human Subjects Are Related to Atherogenesis. Circulating $\mathrm{miR}$ has emerged as a new diagnostic component in cardiovascular diseases $[16,24-26]$. Our previous study using the ApoE-/- mouse model has shown that a repressed level of serum circulating miR-10a is associated with the development of atherosclerosis [13]. Our present study demonstrated that the level of circulating miR-10a in the serum from patients with CAD is significantly lower than that in normal human subjects. These results suggest that low levels of circulating miR-10a in human subjects are related to the development of human $\mathrm{CAD}$, such as atherosclerotic disease.

\section{Conclusions}

Our findings identify the relationship between miR-10a signaling and $\mathrm{CAD}$, i.e., atherosclerosis in humans. Low miR-10a and high GATA6/VCAM-1 levels in the cardiovascular endothelium are highly related to the pathobiology of human atherosclerotic lesions. The reduced expression of endothelial miR-10a seems to enhance the expression of downstream GATA6/VCAM-1 to turn on inflammatory signaling and promote human blood vessels toward atherosclerotic lesions. The expression pattern of low miR-10a and high GATA6/VCAM-1 in cardiovascular endothelium can be helpful as a diagnostic biomarker for atherosclerotic 
lesions. In addition, we also demonstrated that patients with CAD have a significantly lower concentration of miR-10a in their serum compared with healthy subjects. The low serum level of miR-10a can potentially be used to develop new diagnostic strategies for human atherosclerotic diseases. Our study provides novel information regarding miR-10a in relation to human $\mathrm{CAD}$.

\section{Data Availability}

The data used to support the findings of this study are included within the article.

\section{Conflicts of Interest}

The authors declare that there are no conflicts of interest.

\section{Authors' Contributions}

Jong-Tar Kuo, Hsiao-En Tsai, and Ching-Ting Lin contributed equally to this work.

\section{Acknowledgments}

The authors thank Drs. Chien-Sung Tsai, Chih-Yuan Lin, Chih-Cheng $\mathrm{Wu}$, and Li-Jing Chen for collecting human specimens for experiments. This work was supported by the Ministry of Science and Technology, Taiwan (grant numbers MOST-109-2320-B-157-001/108-2320-B-157-001/107-2320B-157-001 (to D.-Y. L.) and MOST-109-2326-B-400-006/ 109-2320-B-400-010-MY3 (to J.-J.C.)).

\section{Supplementary Materials}

Table S1: the characteristics of healthy subjects and CAD patients. (Supplementary Materials)

\section{References}

[1] J.-J. Chiu and S. Chien, "Effects of disturbed flow on vascular endothelium: pathophysiological basis and clinical perspectives," Physiological Reviews, vol. 91, no. 1, pp. 327-387, 2011.

[2] J. Ando and K. Yamamoto, "Vascular mechanobiology endothelial cell responses to fluid shear stress," Circulation Journal, vol. 73, no. 11, pp. 1983-1992, 2009.

[3] S. Chien, "Effects of disturbed flow on endothelial cells," Annals of Biomedical Engineering, vol. 36, no. 4, pp. 554-562, 2008.

[4] J. Zhou, Y.-S. Li, and S. Chien, "Shear stress-initiated signaling and its regulation of endothelial function," Arteriosclerosis, Thrombosis, and Vascular Biology, vol. 34, no. 10, pp. 21912198, 2014.

[5] D.-Y. Lee and J.-J. Chiu, "Atherosclerosis and flow: roles of epigenetic modulation in vascular endothelium," Journal of Biomedical Science, vol. 26, no. 1, p. 56, 2019.

[6] Y. Zhou, H. Huang, and X. Hou, "MicroRNA-133b alleviates hypoxia injury by direct targeting on NOD-like receptor protein 3 in rat H9c2 cardiomyocyte," Cardiology Research and Practice, vol. 2019, Article ID 8092461, 8 pages, 2019.

[7] K. Yang, X. Liu, W. Lin, Y. Zhang, and C. Peng, "Upregulation of microRNA-125b leads to the resistance to inflammatory injury in endothelial progenitor cells," Cardiology Research and Practice, vol. 2020, Article ID 6210847, 7 pages, 2020.

[8] L. J. Chen, S. Y. Wei, and J. J. Chiu, "Mechanical regulation of epigenetics in vascular biology and pathobiology," Journal of Cellular and Molecular Medicine, vol. 17, no. 4, pp. 437-448, 2013.

[9] T. Marin, B. Gongol, Z. Chen et al., "Mechanosensitive microRNAs-role in endothelial responses to shear stress and redox state," Free Radical Biology and Medicine, vol. 64, pp. 61-68, 2013.

[10] J. Zhou, K.-C. Wang, W. Wu et al., "MicroRNA-21 targets peroxisome proliferators-activated receptor- in an autoregulatory loop to modulate flow-induced endothelial inflammation," Proceedings of the National Academy of Sciences, vol. 108, no. 25, pp. 10355-10360, 2011.

[11] Y. Fang, C. Shi, E. Manduchi, M. Civelek, and P. F. Davies, "MicroRNA-10a regulation of proinflammatory phenotype in athero-susceptible endothelium in vivo and in vitro," Proceedings of the National Academy of Sciences, vol. 107, no. 30, pp. 13450-13455, 2010.

[12] D.-Y. Lee, T.-E. Lin, C.-I. Lee et al., "MicroRNA-10a is crucial for endothelial response to different flow patterns via interaction of retinoid acid receptors and histone deacetylases," Proceedings of the National Academy of Sciences, vol. 114, no. 8, pp. 2072-2077, 2017.

[13] D.-Y. Lee, T.-L. Yang, Y.-H. Huang et al., "Induction of microRNA-10a using retinoic acid receptor- $\alpha$ and retinoid $\mathrm{x}$ receptor- $\alpha$ agonists inhibits atherosclerotic lesion formation," Atherosclerosis, vol. 271, pp. 36-44, 2018.

[14] D.-Y. Lee and J.-J. Chiu, "Hemodynamics-based strategy of using retinoic acid receptor and retinoid $\mathrm{X}$ receptor agonists to induce microRNA-10a and inhibit atherosclerotic lesion," Methods in Molecular Biology, vol. 2019, 169 pages, 2019.

[15] A. N. Silahtaroglu, D. Nolting, L. Dyrskjøt et al., "Detection of micrornas in frozen tissue sections by fluorescence in situ hybridization using locked nucleic acid probes and tyramide signal amplification," Nature Protocols, vol. 2, no. 10, pp. 2520-2528, 2007.

[16] S. Fichtlscherer, S. De Rosa, H. Fox et al., "Circulating microRNAs in patients with coronary artery disease," Circulation Research, vol. 107, no. 5, pp. 677-684, 2010.

[17] M. Umetani, C. Mataki, N. Minegishi, M. Yamamoto, T. Hamakubo, and T. Kodama, "Function of GATA transcription factors in induction of endothelial vascular cell adhesion molecule-1 by tumor necrosis factor- $\alpha$, Arteriosclerosis, Thrombosis, and Vascular Biology, vol. 21, no. 6, pp. 917-922, 2001.

[18] J. D. Molkentin, "The zinc finger-containing transcription factors GATA-4, -5, and -6," Journal of Biological Chemistry, vol. 275, no. 50, pp. 38949-38952, 2000.

[19] E. Suzuki, T. Evans, J. Lowry et al., “The HumanGATA-6gene: structure, chromosomal location, and regulation of expression by tissue-specific and mitogen-responsive signals," Genomics, vol. 38, no. 3, pp. 283-290, 1996.

[20] T. Okegawa, R.-C. Pong, Y. Li, and J.-T. Hsieh, "The role of cell adhesion molecule in cancer progression and its application in cancer therapy," Acta Biochimica Polonica, vol. 51, no. 2, pp. 445-457, 2004.

[21] P. Libby, "Inflammation in atherosclerosis," Nature, vol. 420, no. 6917, pp. 868-874, 2002.

[22] K. Iiyama, L. Hajra, M. Iiyama et al., "Patterns of vascular cell adhesion molecule-1 and intercellular adhesion molecule-1 expression in rabbit and mouse atherosclerotic lesions and at sites predisposed to lesion formation," Circulation Research, vol. 85, no. 2, pp. 199-207, 1999. 
[23] I. T. Nizamutdinova, Y. M. Kim, J. I. Chung et al., "Anthocyanins from black soybean seed coats preferentially inhibit TNF- $\alpha$-mediated induction of VCAM- 1 over ICAM-1 through the regulation of GATAs and IRF-1," Journal of Agricultural and Food Chemistry, vol. 57, no. 16, pp. 73247330, 2009.

[24] M. Li, C. Ren, C. Wu, X. Li, X. Li, and W. Mao, "Bioinformatics analysis reveals diagnostic markers and vital pathways involved in acute coronary syndrome," Cardiology Research and Practice, vol. 2020, Article ID 3162581, 11 pages, 2020.

[25] M. S. Ali Sheikh, "Diagnostic role of plasma microRNA-21 in stable and unstable angina patients and association with aging," Cardiology Research and Practice, vol. 2020, Article ID 9093151, 7 pages, 2020.

[26] V. Černá, P. Ostašov, P. Pitule et al., "The expression profile of microRNAs in small and large abdominal aortic aneurysms," Cardiology Research and Practice, vol. 2019, Article ID 8645840, 8 pages, 2019. 